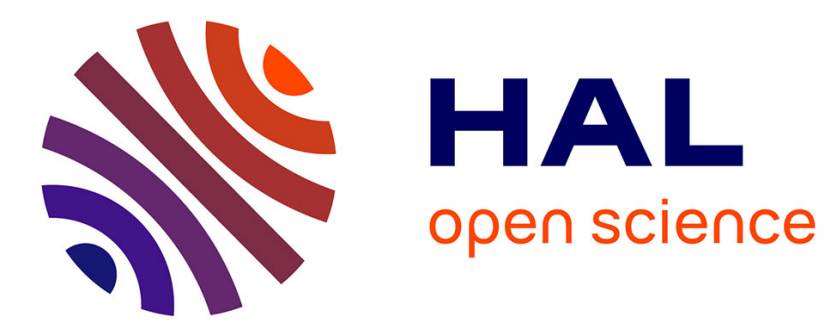

\title{
Synthesis of new bioisosteric hemiasterlin analogues with extremely high cytotoxicity
}

Chinh Pham The, Tuyet Anh Dang Thi, Thi Phuong Hoang, Quoc Anh Ngo,

Duy Tien Doan, Thu Ha Nguyen Thi, Tham Pham Thi, Thu Ha Vu Thi, Mickael Jean, Pierre van de Weghe, et al.

\section{To cite this version:}

Chinh Pham The, Tuyet Anh Dang Thi, Thi Phuong Hoang, Quoc Anh Ngo, Duy Tien Doan, et al. Synthesis of new bioisosteric hemiasterlin analogues with extremely high cytotoxicity. Bioorganic and Medicinal Chemistry Letters, 2014, 24 (22), pp.5216-5218. 10.1016/j.bmcl.2014.09.065 . hal01080910

\section{HAL Id: hal-01080910 \\ https://hal-univ-rennes1.archives-ouvertes.fr/hal-01080910}

Submitted on 7 Nov 2014

HAL is a multi-disciplinary open access archive for the deposit and dissemination of scientific research documents, whether they are published or not. The documents may come from teaching and research institutions in France or abroad, or from public or private research centers.
L'archive ouverte pluridisciplinaire HAL, est destinée au dépôt et à la diffusion de documents scientifiques de niveau recherche, publiés ou non, émanant des établissements d'enseignement et de recherche français ou étrangers, des laboratoires publics ou privés. 


\title{
Synthesis of New Simplified Hemiasterlin Derivatives with $\alpha, \beta$ - Unsaturated Carbonyl Moiety
}

\author{
Chinh Pham The, ${ }^{\mathrm{a}}$ Tuyet Anh Dang Thi, ${ }^{\mathrm{a}}$ Thi Phuong Hoang, ${ }^{\mathrm{a}}$ Quoc Anh Ngo, ${ }^{\mathrm{a}}$ Duy Tien \\ Doan, ${ }^{\mathrm{a}}$ Thu Ha Nguyen Thi, ${ }^{\mathrm{a}}$ Tham Pham Thi, ${ }^{\mathrm{a}}$ Thu Ha Vu Thi, ${ }^{\mathrm{a}}$ M. Jean, ${ }^{\mathrm{b}}$ P. van de Weghe*b \\ and Tuyen Nguyen Van*a \\ ${ }^{a}$ Institute of Chemistry - Vietnam Academy of Science and Technology, 18 Hoang Quoc Viet, Cau Giay, Hanoi, \\ Vietnam. \\ ${ }^{b}$ Equipe Produits Naturels, Synthèses et Chimie Médicinale (PNSCM), UMR CNRS 6226 - Institut des Sciences \\ Chimiques de Rennes, Université de Rennes 1, UFR Sciences Pharmaceutiques et Biologique, 2, Avenue du Prof \\ L. Bernard, F-35043 Rennes Cedex, France.
}

\begin{abstract}
In this article, we report a convenient and efficient method for the synthesis of new simplified derivatives of hemiasterlin in which the $\alpha, \alpha$-dimethylbenzylic moiety $\mathrm{A}$ is replaced by $\alpha, \beta$-unsaturated aryl groups as Michael acceptor. Most of these derivatives have a strong cytotoxic activity on three human tumor cell lines $\left(\mathrm{KB}, \mathrm{Hep}-\mathrm{G}_{2}\right.$ and $\left.\mathrm{MCF}_{7}\right)$. Analogs $\mathbf{1 7 b}$ and $\mathbf{1 7 f}$ showed a high cytotoxicity against $\mathrm{KB}$ and $\mathrm{Hep}-\mathrm{G}_{2}$ cancer cell lines comparable to paclitaxel and ellipticine.
\end{abstract}

Hemiasterlins belong to a family of naturally occurring tripeptides from marine sponges. ${ }^{1}$ The important derivatives of hemiasterlins are hemiasterlin A, hemiasterlin B, and hemiasterlin C, which were isolated from marine sponge Auletta and Cymbastella (Fig. 1) and exhibited potent cytotoxicity in vitro against murine leukemia P388 and human breast, ovarian, colon, and lung cancer cell lines. ${ }^{2,3}$ Hemiasterlins suppress microtubule depolymerization presumably by binding to the vinca-alkaloid binding-domain of tubulin and leading to mitotic arrest and cell death. ${ }^{4 a}$ The synthetic analog HTI-286 (2) displayed especially potent cytotoxicity against paclitaxel $\left(\mathrm{Taxol}^{\mathrm{TM}}\right)$ resistant turmor cell lines in vitro and in vivo and is currently in clinical trials. $^{4 \mathrm{~b}}$
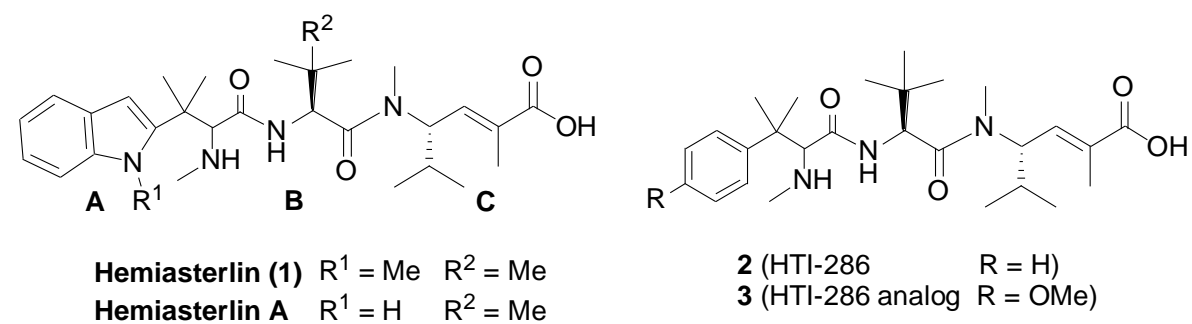

$$
\begin{array}{lll}
\text { Hemiasterlin (1) } & R^{1}=M e & R^{2}=M e \\
\text { Hemiasterlin A } & R^{1}=H & R^{2}=M e \\
\text { Hemiasterlin B } & R^{1}=H & R^{2}=H \\
\text { Hemiasterlin C } & R^{1}=M e & R^{2}=H
\end{array}
$$

Figure 1. Structures of hemiasterlin derivatives and HTI analogs.

There are several reports on the synthesis of new derivatives of hemiasterlin in which the indole aromatic ring in the moiety $\mathbf{A}$ of the original molecule was replaced by aryl functional groups. ${ }^{5,6 a}$ However, asymmetric synthesis of the stereospecific amine group and especially the gem-dimethyl moiety were proved to be highly problematic. To overcome this difficulty, several studies explored modifications of segment $\mathbf{A}$ in which the gem-dimethyl moiety has been eliminated. Some of these derivatives showed promising cytotoxic activity. ${ }^{6}$ 
In recent years, previous works have shown that the presence of a $\alpha, \beta$-unsaturated carbonyl system in peptide derivatives can improve the biological activity compared to the initial compound. ${ }^{7,8}$ Explanations may include either a limitation of free energy of the peptide by addition of a constrained system or by an electrophilic character of the compound leading to the possibility to form covalent bond with the protein target. Based on the idea that hemiasterlin derivatives containing $\alpha, \beta$-unsaturated carbonyl systems could induce a remarkable cytotoxicity, we decided to synthesize new hemiasterlin derivatives in which the $\alpha, \alpha$ dimethylbenzylic group (fragment $\mathrm{A}$ ) is replaced by a $\alpha, \beta$-unsaturated carbonyl group.

New hemiasterlin derivatives were synthesized via classical peptide coupling reactions between two fragments 7a-h and 14a,b. A general procedure for the synthesis of compound $\mathbf{7}$ is outlined in Scheme 1. Compounds 7 were prepared from glycine in three steps. ${ }^{9}$ The synthesis started by acetylation of glycine with acetic anhydride in water at room temperature following by condensation with aryl aldehydes using sodium acetate in the presence of acetic anhydride at $90{ }^{\circ} \mathrm{C}$ for $12 \mathrm{~h}$ which afforded azalactones $\mathbf{6 a - h}$ in $72-83 \%$ yields. ${ }^{9}$ Finally, azalactones $\mathbf{6 a - h}$ were hydrolyzed in aqueous sodium hydroxide, followed by treatment with hydrochloric acid $(12 \mathrm{~N})$ at $100{ }^{\circ} \mathrm{C}$ for $4 \mathrm{~h}$ to give compounds $\mathbf{7 a - h}$ in $65-88 \%$ yields.

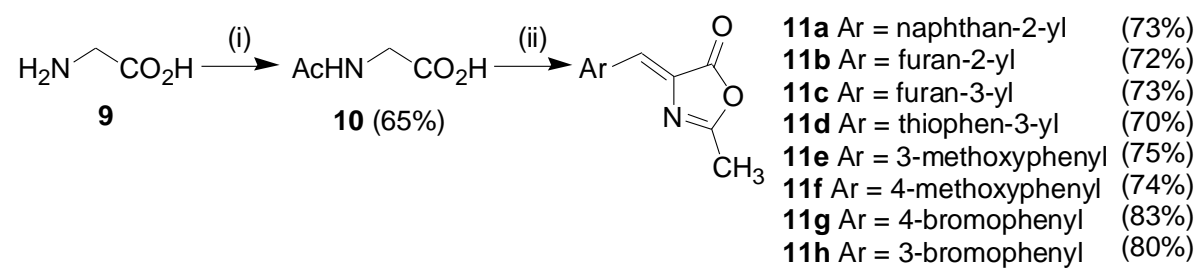

\footnotetext{
(iii) $\mathrm{Ar} \mathrm{CO}_{2} \mathrm{H} \mathrm{12a}, 82 \%-12 \mathrm{~b}, 80 \%-12 \mathrm{c}, 75 \%$

NHAc $12 \mathrm{~d}, 65 \%-12 \mathrm{e}, 85 \%-12 \mathrm{f}, 83 \%$
}

Scheme 1. Reagents and conditions (i) 2.0 equiv $\mathrm{Ac}_{2} \mathrm{O}, \mathrm{H}_{2} \mathrm{O}$, rt, $20 \mathrm{~h}$. (ii) 0.75 equiv $\mathrm{ArCHO}$, 1.0 equiv $\mathrm{AcONa}, \mathrm{Ac}_{2} \mathrm{O}, 90^{\circ} \mathrm{C}, 12 \mathrm{~h}$. (iii) $\mathrm{NaOH}(1 \mathrm{~N})$ then $\mathrm{HCl}(12 \mathrm{~N}), 100{ }^{\circ} \mathrm{C}, 4 \mathrm{~h}$.

Compounds 14a,b were obtained from Boc-L-valine in 6 steps as depicted in Scheme 2. ${ }^{4}$ In the first step, Boc-L-valines 8a,b were converted to Weinreb amide 9a,b in good yields (86 and $81 \%$ ) by treatment with a mixture of $N, O$-dimethylhydroxylamine hydrochloride, 1-ethyl-3-(3dimethylaminoproryl)carbodiimide (EDC) and hydroxybenzotriazole (HOBt) in the presence of N-ethylisopropylamine ( $i$-PrNHEt) at room temperature for $12 \mathrm{~h} \cdot{ }^{10}$ Reduction of $\mathbf{9 a , b}$ using $\mathrm{LiAlH}_{4}$ in THF was carried out at room temperature for $1 \mathrm{~h}$ to give aldehydes $\mathbf{1 0 a}, \mathbf{b}$ in $78 \%$ and $65 \%$ yields, respectively. ${ }^{11}$ Afterward, Wittig reaction of aldehydes $\mathbf{1 0 a , b}$ with ethyl 2(triphenylphosphoranylidene)propanoate was carried out at reflux in $\mathrm{CH}_{2} \mathrm{Cl}_{2}$ for $6 \mathrm{~h}$ to afford the alkenoates $11 \mathbf{a}$ and $\mathbf{1 1 b}$ in $86 \%$ and $88 \%$ yields, respectively. ${ }^{6,12}$ Then, removal of the Boc group using trifluoroacetic acid in $\mathrm{CH}_{2} \mathrm{Cl}_{2}$ at room temperature for $1 \mathrm{~h}$ led to 12a,b in high yields (90 and 95\%). The expected compounds $\mathbf{1 4 a}, \mathbf{b}$ are finally obtained after coupling $\mathbf{1 2 a}, \mathbf{b}$ and Boc-L-leucine in the presence of EDC/HOBt in DMF at room temperature for $12 \mathrm{~h}$ following by Boc deprotection in classical reaction conditions. 


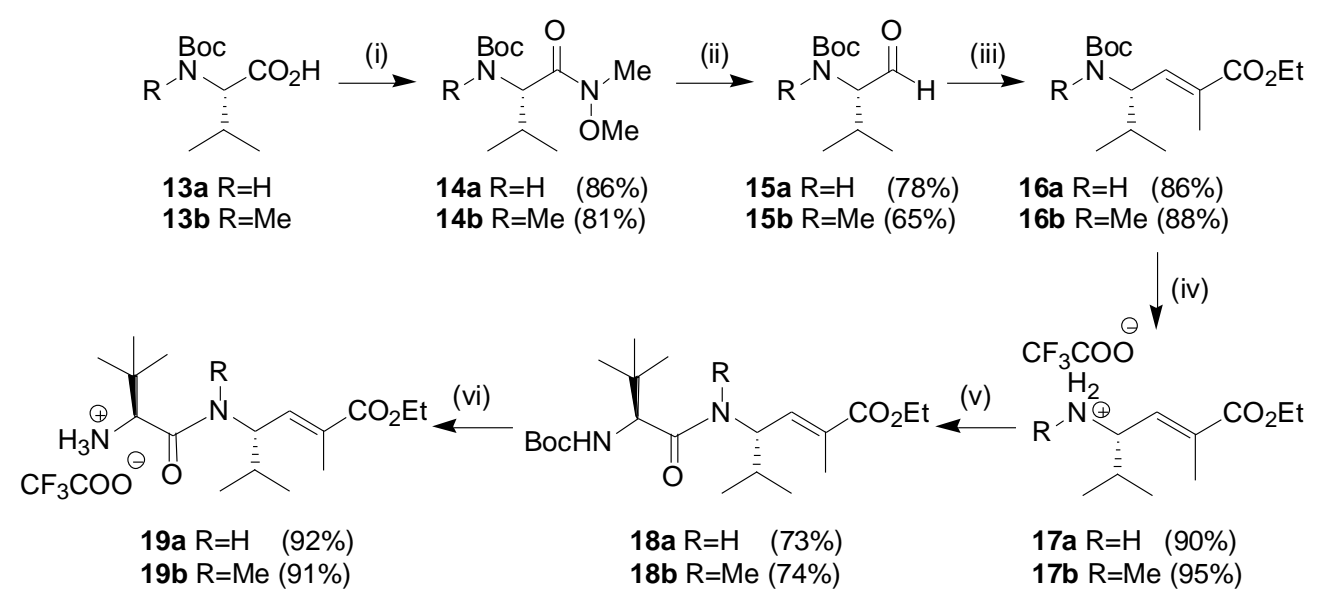

Scheme 2. Reagents and conditions (i) 1.1 equiv $\mathrm{NH}(\mathrm{Me}) \mathrm{OMe}, 1.1$ equiv EDC, 1.1 equiv HOBt, 2.0 equiv $i$-PrNHEt, DMF, rt, $12 \mathrm{~h}$. (ii) 4.0 equiv LAH, THF, rt, $1 \mathrm{~h}$. (iii) $\mathrm{Ph}_{3} \mathrm{P}=\mathrm{C}\left(\mathrm{CH}_{3}\right) \mathrm{CO}_{2} \mathrm{Et}, \mathrm{CH}_{2} \mathrm{Cl}_{2}$, reflux, 6 h. (iv) TFA, $\mathrm{CH}_{2} \mathrm{Cl}_{2}$, rt, 1 h. (v) 1.0 equiv Boc-LLeucine, 1.1 equiv EDC, 1.1 equiv HOBt, 2.0 equiv $i$-PrNHEt, DMF, rt, 12 h. (vi) TFA, $\mathrm{CH}_{2} \mathrm{Cl}_{2}$, $\mathrm{rt}, 1 \mathrm{~h}$.

The final compounds 16a-h were then prepared in two steps after peptide coupling reactions of 14a with amides 7a-h following by the saponification of ester using a $1 \mathrm{~N}$ lithium hydroxide solution (Scheme 3-a). Similarly, the hemiasterlin derivatives 18a-g were obtained from 14b (Scheme 3-b).
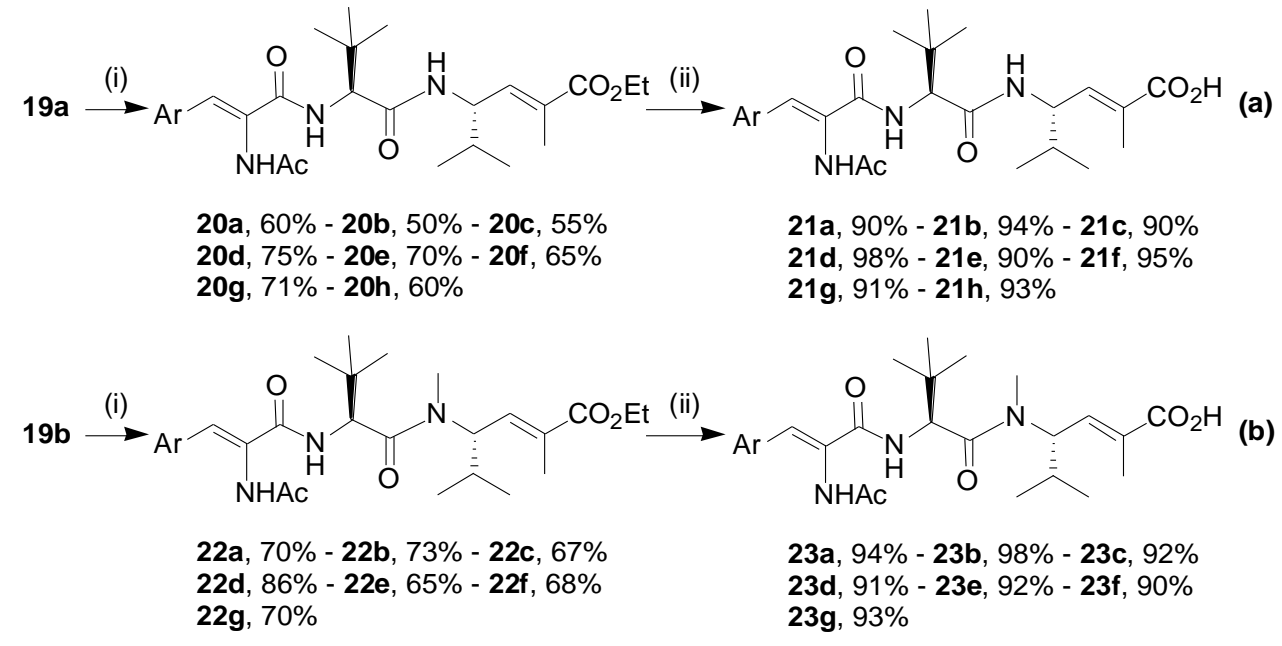

Scheme 3. Reagents and conditions (i) 1.0 equiv 12a-h, 1.1 equiv EDC, 1.1 equiv HOBt, 2.0 equiv $i$-PrNHEt, DMF, rt, 12 h. (ii) 10 equiv $\mathrm{LiOH}, \mathrm{MeOH}: \mathrm{H}_{2} \mathrm{O}(2: 1)$, rt, $10 \mathrm{~h}$.

All compounds 15a-h, 16a-h, 17a-g and 18a-g were evaluated in vitro for their cytotoxic activity against four human tumor cell lines $\left(\mathrm{KB}, \mathrm{Hep}-\mathrm{G}_{2}, \mathrm{LU}\right.$ and $\left.\mathrm{MCF}_{7}\right)$ and the results were summarized in Table 1. Eleven hemiasterlin derivatives showed strong activity against the KB, Hep- $\mathrm{G}_{2}$ cell line with $\mathrm{IC}_{50}$ value below $100 \mathrm{nM}$. Analogs $\mathbf{1 6 b}, \mathbf{1 7 b}$ and $\mathbf{1 8 f}$ exhibited potent cytotoxicity against the $\mathrm{KB}$ cell line with $\mathrm{IC}_{50}=8.2,3.5$ and $3.7 \mathrm{nM}$, respectively. Meanwhile analogs $\mathbf{1 6 b}, \mathbf{2 2 1 7}$ and $\mathbf{1 7 f}$ displayed potent cytotoxicity against the $\mathrm{Hep}-\mathrm{G}_{2}$ cell line with $\mathrm{IC}_{50}$ value 17.8, 16.3 and $3.7 \mathrm{nM}$, respectively. Derivatives 15a, 15e and 17a presented a cytotoxic activity against $\mathrm{MCF}_{7}$ cell line with $\mathrm{IC}_{50}$ value 42.3, 60.0 and $67.9 \mathrm{nM}$. Concerning the last cell line, the LU cell line, the hemiasterlin analogs showed weak activities with $\mathrm{IC}_{50}$ values above 
$269 \mathrm{nM}$. It is noteworthy to mention that two derivatives $\mathbf{1 7} \mathbf{a}$ and $\mathbf{1 7 f}$ present a cytotoxic activity against two cancer cell lines $\left(\mathrm{KB}, \mathrm{Hep}-\mathrm{G}_{2}\right)$ comparable with those of ellipticine and paclitaxel

Table 1. Cytotoxicity evaluation.

\begin{tabular}{|c|c|c|c|c|c|}
\hline \multirow[b]{2}{*}{ Entry } & \multirow[b]{2}{*}{ compound } & \multicolumn{4}{|c|}{$\mathrm{IC}_{50}(\mathrm{nM})$} \\
\hline & & $\mathrm{KB}$ & $\begin{array}{c}\text { Hep- } \\
\mathrm{G}_{2}\end{array}$ & LU & $\mathrm{MCF}_{7}$ \\
\hline 1 & $15 \mathbf{a}$ & 30.8 & 39.7 & $>269$ & 42.3 \\
\hline 2 & $15 b$ & 160.5 & 214.2 & $>269$ & $>269$ \\
\hline 3 & $15 c$ & 86.2 & 67.3 & $>269$ & $>269$ \\
\hline 4 & $15 d$ & 107.4 & 57.6 & $>269$ & 203 \\
\hline 5 & $15 e$ & 77.4 & 47.7 & 228.4 & 62 \\
\hline 6 & $15 f$ & $>269$ & $>269$ & $>269$ & $>269$ \\
\hline 7 & $15 \mathrm{~g}$ & $>269$ & $>269$ & $>269$ & $>269$ \\
\hline 8 & $15 \mathrm{~h}$ & $>269$ & $>269$ & $>269$ & $>269$ \\
\hline 9 & $6 \mathbf{a}$ & 63 & 33.4 & $>269$ & $>269$ \\
\hline 10 & 6b & 8.2 & 17.8 & $>269$ & $>269$ \\
\hline 11 & $6 c$ & 155.4 & $>269$ & $>269$ & $>269$ \\
\hline 12 & $6 d$ & $>269$ & $>269$ & $>269$ & $>269$ \\
\hline 13 & $6 e$ & $>269$ & $>269$ & $>269$ & $>269$ \\
\hline 14 & $6 f$ & $>269$ & $>269$ & $>269$ & $>269$ \\
\hline 15 & $6 \mathrm{~g}$ & $>269$ & $>269$ & $>269$ & $>269$ \\
\hline 16 & $6 h$ & $>269$ & $>269$ & $>269$ & $>269$ \\
\hline 17 & $17 \mathbf{a}$ & 23.0 & 23.1 & $>269$ & 67.9 \\
\hline 18 & $17 b$ & 3.5 & 31.4 & 231.0 & 178.4 \\
\hline 19 & $17 \mathrm{c}$ & 24.1 & 16.3 & 158 & 112.8 \\
\hline 20 & 17d & 202 & 15.0 & $>269$ & $>269$ \\
\hline 21 & $17 \mathrm{e}$ & $>269$ & $>269$ & $>269$ & $>269$ \\
\hline 22 & $17 f$ & 3.7 & 3.7 & $>269$ & 215 \\
\hline 23 & $17 \mathrm{~g}$ & 49.0 & $>269$ & $>269$ & $>269$ \\
\hline 24 & $18 \mathbf{a}$ & 69.7 & 13.0 & $>269$ & $>269$ \\
\hline 25 & $18 b$ & 188.0 & $>269$ & $>269$ & $>269$ \\
\hline 26 & $18 \mathrm{c}$ & $>269$ & 149.4 & $>269$ & $>269$ \\
\hline 27 & 18d & 234 & 63.2 & $>269$ & $>269$ \\
\hline 28 & $18 \mathrm{e}$ & $>269$ & $>269$ & $>269$ & $>269$ \\
\hline 29 & $18 f$ & 15.8 & 63.8 & $>269$ & $>269$ \\
\hline 30 & $18 g$ & $>269$ & $>269$ & $>269$ & $>269$ \\
\hline 31 & Ellipticine & 1.26 & 1.26 & 1.82 & 2.15 \\
\hline 32 & Paclitaxel & 3.9 & 0.19 & - & - \\
\hline
\end{tabular}

In conclusion, a concise synthetic approach for new modified hemiasterlin derivatives was achieved in which the $\alpha, \alpha$-dimethylbenzylic group and amino NHMe moiety were replaced respectively by a $\alpha, \beta$-unsaturated aryl and an amide $\mathrm{NH}$-Ac group avoiding the synthesis of the chiral fragment $\mathbf{A}$. Most of these derivatives possess strong cytotoxic activity on three human tumor cell lines (KB, Hep- $\mathrm{G}_{2}$ and $\left.\mathrm{MCF}_{7}\right)$ and two analogs, $\mathbf{1 7 b}$ and $\mathbf{1 7 f}$, showed a comparable cytotoxicity activity to paclitaxel and ellipticine against $\mathrm{KB}$ and $\mathrm{Hep}-\mathrm{G}_{2}$ cancer cell lines. Based on previously reported work, ${ }^{6 \mathrm{~b}}$ we can envisage that our hemiasterlin derivatives act as tubulin 
polymerization inhibitors. From our best compounds, more detailed biological studies will be undertaken and will be reported in due course.

\section{Acknowledgments}

This work was financially supported in part by scientific research and technological development project (code: ĐT.NCCB-ĐHUD.2011-G/07).

\section{Supplementary Material}

Supplementary data associated with this article can be found, in the online version, at doi:

\section{References and notes}

1. Talpir, R.; Benayahu, Y.; Kashman, Y.; Pannell, L.; Schleyer, M. Tetrahedron Lett., 1994, 35, 4453.

2. Gamble, W. R.; Durso, N. A.; Fuller, R. W.; Westergaard, C. K.; Johnson, T. R.; Sackett, D. L.; Hamel, E.; Cardellina II, J. H.; Boyd, M. R. Bioorg. Med. Chem., 1999, 7, 1611.

3. Coleman, J. E.; Silva, E. D.; Kong, F.; Andersen, R. J.; Allen, T. M. Tetrahedron, 1995, $51,10653$.

4. (a) Anderson, H. J.; Coleman, J. E.; Andersen, R. J.; Roberge, M. Cancer Chemother. Pharmacol., 1997, 39, 223. (b) Loganzo, F.; Discafani, C. M.; Annable, T.; Beyer, C.; Musto, S.; Hari, M.; Tan, X.; Hardy, C.; Hernandez, R.; Baxter, M.; Singanallore, T.; Khafizova, G.; Poruchynsky, M. S.; Fojo, T.; Nieman, J. A.; Ayral-Kaloustian, S.; Zask, A.; Andersen, R. J.; Greenberger, L. M. Cancer Res., 2003, 63, 1838-1845.

5. (a) Andersen, R.; Piers, E.; Nieman, J.; Coleman, J.; Roberge, M. WO9932509. (b) Yamashita, A.; Norton, E. B.; Kaplan, J. A.; Niu, C.; Loganzo, F.; Hernand, R.; Beyer, C. F.; Annable, T.; Musto, S.; Discafani, C.; Zask, A.; Ayral-Kaloustian, S. Bioorg. Med. Chem. Lett., 2004, 14, 5317. (c) Greenberger, L. M.; Loganzo, F. Jr; DiscafaniMarro, C.; Zask, A.; Ayral-Kaloustian, S. WO2004026293. (d) Mokale, S. N.; Lokwani, D.; Shinde, D. Bioorg. Med. Chem., 2012, 20, 3119. (d) Mali, S. M.; Bandyopadhyay, A.; Jadhav, S. V.; Kumar, M. G.; Gopi, H. N. Org. Biomol. Chem., 2011, 9, 6566.

6. (a) Niew, J. A.; Coleman, J. E.; Wallace, D. J. ; Piers, E.; Lim, L. Y.; Roberge, M.; Andersen, R. J. J. Nat. Prod., 2003, 6, 183. (b) Simoni, D.; Lee, R. M.; Durrant, D.; Chi, N. W.; Baruchello, R.; Rondanin, R.; Rullo, C.; Marchetti, P. Bioorg. Med. Chem. Lett., 2010, 20, 3431. (c) Murray, W. V.; Sun, S.; Turchi, I. J.; Brown, F. K.; Gauthier, A. D. J. Org. Chem., 1999, 64, 5930. (d) Grison, C.; Geneve, S.; Halbin, E.; Coutrot, P. Tetrahedron, 2001, 57, 4903.

7. Yamazaki, Y.; Tanaka, K.; Nicholson, B.; Deyanat-Yazdi, G.; Pott, B.; Yoshida, T.; Oda, A.; Kitagawa, T.; Orikasa, S.; Kiso, Y.; Yasui, H.; Akamatsu, M.; Chinen, T.; Usui, T.; Shinozaki, Y.; Yakushiji, F.; Miller, B. R.; Neuteboom, S.; Palladino, M.; Kanoth, K.; Lloyd, G. K.; Hayashi, Y. J. Med. Chem., 2012, 55, 1056.

8. Pijus, K. M.; Zhiyong, R.; Xiaomin, C.; Chiyi, X.; McMurray, J. S. J. Med. Chem., 2009, 52, 6126.

9. (a) Herbst, R. M. ; Shemin, D. Org. Synth., 1939, 19, 1. (b) Thomas, C.; Jodie, B.; Flavio, C. Tetrahedron Lett., 2010, 51, 625. (c) Mesaik, M. A.; Rahat, S.; Khan, K. M.; Zia-Ullah; Choudhary, M. I.; Murad, S.; Ismail, Z.; Atta-ur-Rahman; Ahmad, A. Bioorg. Med. Chem., 2004, 12, 2049. (d) RajanBabu, T. V.; Ayers, T. A.; Halliday, G. A.; You, K. K.; Calabrese, J. C. J. Org. Chem., 1997, 62, 6012. (e) Cutolo, M.; 
Fiandanese, V.; Naso, F.; Sciacovelli, O. Tetrahedron Lett., 1983, 24, 4603. (f) Baell, J. B.; Garrett, T. P. J.; Hogarth, P. M.; Mattews, B. R.; McCarthy, T. D.; Pietersz, G. A. WO2000015214. (g) Sawant, S. D.; Barvrkar, A. A.; Chabukswar, A. R.; Sarak, S. D. Journal of Applicable Chemistry, 2013, 2, 372. (h) Blanco-Lomas, M.; Funes-Ardoiz, I.; Campos, P. J.; Sampedro, D. Eur. J. Org. Chem., 2013, 6611. (i) Funes-Ardoiz, I.; Blanco-Lomas, M.; Campos, P. J.; Sampedro, D. Tetrahedron, 2013, 69, 9766.

10. (a) Taillier, C.; Bellosta, V.; Meyer, C.; Cossy, J. Org. Lett., 2004, 6, 2145. (b) Nahm, S.; Weinreb, S. M. Tetrahedron Lett., 1981, 39, 3815. (c) Tyrrell, E.; Brawn, P.; Carew, M.; Greenwood, I. Tetrahedron Lett., 2011, 52, 369. (d) Kim, J. G.; Jang, D. O. Bull. Korean Chem. Soc., 2010, 31, 171.

11. (a) Angelastro, M. R.; Mehdi, S.; Burkhart, J. P.; Peet, N. P.; Bey, P. J. Med. Chem., 1990, 33, 11. (b) Burke, A. J.; Davies, S. G.; Garner, C. A.; McCarthy, T. D.; Roberts, P. M.; Smith, A. D.; Rodriguez-Solla, H.; Vicker, R. J. Org. Biomol. Chem., 2004, 2, 1387.

12. Murphy, J. A.; Commeueuc, A. G. J.; Snaddon, T. N.; McGuire, T. M.; Khan, T. A.; Hisler, K.; Dewis, M. L.; Carling, M. Org. Lett., 2005, 7, 1427. 